INPLASY

PROTOCOL

To cite: Yu et al. Efficacy and safety of Tripterygium Wilfordii Polyglycosides for Idiopathic membranous nephropathy : An overview of systematic reviews and meta-analyses. Inplasy protocol 202210088. doi: 10.37766/inplasy2022.1.0088

Received: 17 January 2022

Published: 17 January 2022

Corresponding author: Haiyan Yu

960971062@qq.com

Author Affiliation:

Changchun University of Chinese Medicine.

Support: Self-financing.

Review Stage at time of this submission: Preliminary searches.

Conflicts of interest:

None declared.

\section{Efficacy and safety of Tripterygium Wilfordii Polyglycosides for Idiopathic membranous nephropathy : An overview of systematic reviews and meta-analyses}

\author{
Yu, HY1; Zhang, SL2; Chang, TY3; Liu, MX².
}

Review question / Objective: This review aims to assess and evaluate the evidence of the treatment with Tripterygium Wilfordii Polyglycosides (TWP) on individuals with Idiopathic membranous nephropathy (IMN. A cohort study was selected.

Condition being studied: Plenty of trials evaluating the efficacy and safety of the treatment with Tripterygium Wilfordii Poly-glycoside (TWP) on Idiopathic membranous nephropathy (IMN), along with numerous pharmacological studies on the active ingredient of Tripterygium Wilfordii have been published in recent years. TWP may be a potential effective drug for the treatment of iMN, which needs the support from the evidence-based traditional medicine study.

INPLASY registration number: This protocol was registered with the International Platform of Registered Systematic Review and Meta-Analysis Protocols (INPLASY) on 17 January 2022 and was last updated on 17 January 2022 (registration number INPLASY202210088).

\section{INTRODUCTION}

Review question / Objective: This review aims to assess and evaluate the evidence of the treatment with Tripterygium Wilfordii Polyglycosides (TWP) on individuals with Idiopathic membranous nephropathy (IMN). A cohort study was selected.
Condition being studied: Plenty of trials evaluating the efficacy and safety of the treatment with Tripterygium Wilfordii Polyglycoside (TWP) on Idiopathic membranous nephropathy (IMN), along with numerous pharmacological studies on the active ingredient of Tripterygium Wilfordii have been published in recent years. TWP may be a potential effective drug for the treatment of iMN, which needs the support 
from the evidence-based traditional medicine study.

\section{METHODS}

Participant or population: The participants of this study were individuals with IMN, regardless of staging of the disease.

Intervention: The intervention of this study was treatment with TWP, without any restriction on dosage and duration.

Comparator: There is no limitation on the treatment of the control group, which can be routine therapy.

Study designs to be included: Randomized controlled study.

Eligibility criteria: Efficiency: After treatment, the symptoms were significantly improved, and $24 \mathrm{~h}$ urinary albumin returned to normal level, or 24-hour urinary protein was decreased over $50 \%$. This is the effective target. Safety:After treatment, the abnormal blood routine, abnormal liver function and infection rate were counted.This is the safety target.

Information sources: PubMed, the Cochrane Library, China National Knowledge Infrastructure Database (CNKI), Chinese Biomedical Literature Database (CBM), Wan Fang Database, China Science and Technology Journal Database (VIP).

Main outcome(s): PRO:24h urine protein; After treatment, the symptoms were significantly improved, and $24 \mathrm{~h}$ urinary albumin returned to normal level, or 24hour urinary protein was decreased over $50 \%$. This is the effective target. After treatment, the abnormal blood routine, abnormal liver function and infection rate were counted.This is the safety target.

Quality assessment / Risk of bias analysis: The reporting and methodological quality of the included systematic reviews and meta-analyses will be assessed according to AMSTAR-2 and PRISMA statement.3 people will be involved in the quality assessment. when Haiyan $\mathrm{Yu}$ has disagreement with Tianying Chang, another senior doctor (Shoulin Zhang) make the final judgment and decision.

Strategy of data synthesis: Design data extraction table according to Pico principle, extract the basic information of the included literature. Effective rate, PRO, Scr, BUN, ALB, ALT, AST, TG, CHO,WBC,HGB,PLT will be synthesised. risk ratios for individual studies will be combined using a random effects metaanalysis.

Subgroup analysis: There is no limitation on the treatment of the control group, which can be routine therapy. Details of the planned analytic : $x 2$ test was used to test the heterogeneity among the studies. If the heterogeneity test showed that there was no heterogeneity among the studies or the heterogeneity was small( $\left(^{2} 0.1\right)$, select the fixed effect analysis model; if $\left(I^{2} \geq 50 \%\right.$, $P \leq 0.1)$, elect the random effects analysis model.

Sensitivity analysis: If there is obvious heterogeneity between a group of studies, the reasons for the heterogeneity should be explored from multiple aspects, such as the characteristics of the research object, the degree of variation of intervention measures, and sensitivity analysis or subgroup analysis should be conducted when necessary to explain the heterogeneity.

Language: English and Chinese.

Country(ies) involved: China.

Keywords: Tripterygium Wilfordii Polyglycosides,Idiopathic membranous nephropathy,metaanalysis, Systematic Review.

Contributions of each author:

Author 1 - Haiyan Yu.

Author 2 - Shoulin Zhang.

Author 3 - Tianying Chang.

Author 4 - Meixi Liu. 